\title{
Genetic regulation of wheat inflorescence development
}

Oxana B. Dobrovolskaya

VNIIKR, Moscow region, Ramenskoe distinct, Bykovo, Russia

ICG SB RAS, Novosibirsk, Russia, oxanad@bionet.nsc.ru
Alina E. Dresvyannikova

ICG SB RAS, Novosibirsk, Russia

alinka.dresvyannikova@gmail.com
Petr Martinrk Agrotest Fyto, Ltd, Kroměříž, Czech Republic martinek@vukrom.cz

\begin{abstract}
Identification of genetic factors that control inflorescence development of important crops and study on their structure and functions have fundamental and practical importance. The obtained results showed that different genetic loci take part in the spikelet meristem determinacy of the genuine branching and false-true spike ramification wheat nonstandard morphotypes.
\end{abstract}

Keywords - wheat, non-standard spike morphotypes, inflorescence development, meristem identity, meristem determinacy

\section{Motivation and aim}

\section{Motivation}

Investigation of the structural and functional organization of genetic determinants of plant development is an actual problem of modern plant genetics and developmental biology. Aim

The aim of this work is to study on genetic regulation of wheat spikelet and floret meristem activities, namely, the establishing of identity and determinacy of wheat inflorescence meristems.

\section{Methods}

Accessions with abnormalities in spike development from the ICG SB RAS wheat collection served as a unique experimental model in this research. A set of classical and modern approaches of genetics and developmental biology, including high-throughput genotyping, light and electron microscopy methods and modern bioinformatic approaches was used.

\section{Results}

Using DArTseq genotyping, wheat chromosome maps (genome BBAA) were constructed, quantitative trait loci (QTLs) that determine the formation of non-standard spike morphotypes associated with abnormalities in the establishing of identity and determinacy of inflorescence meristems were identified. The formation of the false true spike ramification morphotype is controlled by the qshr2-2A locus of chromosome $2 \mathrm{AL}$, and by two loci (chr.7A) with minor effects. It was found that, along with the $w f z p-A . B h 1$ gene, the formation of additional spikelets of the wheat genuine branching morphotype is influenced by QTLs on chromosomes 7AL and 5BL. Therefore, different genetic loci take part in determinacy of the spikelet meristem of the genuine branching and the false-true spike ramification morphotypes.

\section{ACKNOWLEDGMENT}

This work was supported by the RFBR (18-04-00483). 